\title{
Reactivity of allergy skin test in healthy volunteers
}

\author{
Phisit Supakthanasiri ${ }^{1}$, MD, Jettanong Klaewsongkram $^{1}$, MD, Hiroshi Chantaphakul ${ }^{1}$, MD
}

INTRODUCTION Healthy individuals may be exposed and sensitised to allergens, and have a positive response to a skin prick test despite being asymptomatic. The objectives of this study were to evaluate the prevalence of atopic sensitisation and identify the reactivity of healthy volunteers to common aeroallergens.

METHODS Healthy volunteers with no known allergic symptoms were recruited in this study. All volunteers were scheduled to undergo a skin prick test with 16 common aeroallergens that were previously identified among atopic patients.

RESULTS A total of 100 volunteers (mean age 28 years) were enrolled in this study. 42 volunteers had positive skin prick tests for at least one allergen. The median number of sensitised allergen was 2 (range 1-7). Volunteers with positive skin tests $(n=42)$ were younger than those with negative skin tests $(n=58)$ (mean age 25.5 vs. 29.2 years; $p<0.05)$. The group with positive skin tests also had a higher proportion of males $(57.1 \%$ vs. $31.0 \% ; p<0.01)$ and firstdegree relatives with a history of atopic diseases ( $31.0 \%$ vs. $10.3 \% ; p<0.05)$. The most common sensitised allergens in these healthy asymptomatic volunteers were mite $(n=33)$, house dust $(n=23)$ and American cockroach $(n=20)$.

CONCLUSION In this study, up to $42 \%$ of healthy volunteers, particularly those with a family history of atopy, were sensitised to allergens. Reactivity of the skin test without allergic symptoms, however, does not indicate allergic disease. Therefore, the skin test should only be indicated in atopic symptomatic individuals.

Keywords: allergic rhinitis, allergy, asthma, prick test, sensitivity

\section{INTRODUCTION}

There is a rise in the prevalence of common allergic diseases, including asthma, allergic rhinitis, atopic dermatitis, food allergy and anaphylaxis, especially in developing countries. ${ }^{(1-4)}$ Lifestyle and environmental exposure is considered to be one of the major contributing factors for this increase. Exposure to various common allergens could cause sensitisation of the immune system, leading to the activation of specific immunoglobulin E (IgE) production. Activation of IgE can be evaluated using a skin prick test. It is important to use allergens that are relevant to a person's environment, as the sensitisation pattern may differ across regions. Therefore, the allergens used in skin prick tests for allergic patients should be the allergens common to the region in which the patient developed the allergy. Healthy individuals may have a positive response to a skin prick test if they were exposed to an allergen in the past and became sensitised to it; however, they may not have allergic disease if they are asymptomatic, although they may be at risk of developing symptoms in the future. ${ }^{(5-7)}$

In Thailand, studies of allergen skin tests in healthy subjects are usually conducted among control populations. ${ }^{(8-10)}$ As these studies had a small number of controls and used few allergenic extracts, the current study thus aimed to estimate the prevalence of atopic sensitisation and identify the common aeroallergens healthy volunteers react to.

\section{METHODS}

Healthy volunteers with no known allergic symptoms of chronic rhinitis or asthma were recruited in this cross-sectional study. The majority of the volunteers were medical personnel of Chulalongkorn University Hospital, Thailand, and family members of patients with allergies seen in the hospital. All volunteers were briefed in detail on the skin prick test and its potential side effects. Written informed consent was obtained from the volunteers. The study was approved by the institutional ethics committee of Chulalongkorn University Hospital, and all procedures conducted conformed to institutional guidelines. Volunteers were excluded from the study if they were younger than 18 years of age, had received antihistamine within three days prior to the skin test, or were pregnant.

After completing the baseline demographic data form and questionnaire, the volunteers were scheduled to undergo a skin prick test. A total of 16 aeroallergens that had previously been identified as common allergens among atopic patients in Thailand $^{(9,11)}$ were used in our study. These allergens were standardised Bermuda grass, standardised Timothy grass, Acacia spp., Alternaria tenuis, dog epithelia (mixed breeds), standardised cat dander, kapok seeds, Orris root, pyrethrum, house dust, Dermatophagoides pteronyssinus (i.e. mite), American cockroach, national weed mix, mixed feathers, mould mix and Aspergillus mix (Greer Laboratories, Lenoir, NC, USA). The diameters

${ }^{1}$ Division of Allergy and Immunology, Department of Medicine, Faculty of Medicine, Chulalongkorn University, Bangkok, Thailand

Correspondence: Dr Hiroshi Chantaphakul, Assistant Professor, Division of Allergy and Immunology, Department of Medicine, Faculty of Medicine, Chulalongkorn University, 1873 Rama 4 Road, Patumwan District, Bangkok 10330, Thailand. hchantaphakul@gmail.com 
Table I. Baseline characteristics and skin prick test results of the study population.

\begin{tabular}{|c|c|c|c|}
\hline \multirow[t]{2}{*}{ Characteristic } & \multicolumn{2}{|c|}{ Skin prick test result } & \multirow{2}{*}{$\begin{array}{c}\text { Total } \\
(n=100)\end{array}$} \\
\hline & Positive $(n=42)$ & Negative $(n=58)$ & \\
\hline Age $^{*, \S}$ (yrs) & $25.5(19-51)$ & $29.2(19-59)$ & $28.0(19-59)$ \\
\hline \multicolumn{4}{|l|}{ Gender,"ף } \\
\hline Male & 24 & 18 & 42 \\
\hline Female & 18 & 40 & 58 \\
\hline \multicolumn{4}{|l|}{ Family history of atopy $y^{+, \S}$} \\
\hline Yes & 13 & 6 & 19 \\
\hline No & 29 & 52 & 81 \\
\hline No. of positive allergens ${ }^{*}$ & $2(1-7)$ & - & $0(0-7)$ \\
\hline Histamine wheal diameter* $(\mathrm{mm})$ & $5.20(3.5-7.5)$ & $5.28(3.75-8.5)$ & $5.24(3.5-8.5)$ \\
\hline
\end{tabular}

${ }^{*}$ Data is presented as mean (range). ${ }^{\dagger}$ Data is presented as no. of volunteers. ${ }^{\ddagger}$ Data is presented as median (range). ${ }^{\S} \mathrm{p}<0.05$ when compared between positive and negative skin prick test results. ${ }^{9} p<0.01$ when compared between positive and negative skin prick test results.

Table II. Volunteers with positive skin prick test results for each aeroallergen.

\begin{tabular}{lccccc}
\hline Aeroallergen & \multicolumn{5}{c}{ No. of volunteers } \\
\cline { 2 - 6 } & Total & $\mathbf{1 +}$ & $\mathbf{2 +}$ & $\mathbf{3 +}$ & $\mathbf{4 +}$ \\
\hline House dust & 23 & 4 & 7 & 5 & 7 \\
Mite & 33 & 3 & 15 & 4 & 11 \\
Alternaria tenuis & 4 & - & 4 & - & - \\
Aspergillus mix & 1 & - & 1 & - & - \\
Mould mix & 1 & - & 1 & - & - \\
Bermuda grass & 2 & - & 1 & 1 & - \\
Timothy grass & - & - & - & - & - \\
Cat dander & 4 & - & 1 & 1 & 2 \\
Dog epithelia & 3 & - & 1 & 2 & - \\
Kapok seeds & 3 & - & 2 & 1 & - \\
American cockroach & 20 & 2 & 15 & 3 & - \\
Mixed feathers & 1 & 1 & - & - & - \\
Acacia spp. & 2 & - & 2 & - & - \\
Orris root & 2 & - & 2 & - & - \\
National weed mix & 5 & 1 & 3 & 1 & - \\
Pyrethrum & - & - & - & - & - \\
\hline $1+$ : & 2 & & & -
\end{tabular}

1+: diameter < half of histamine diameter; $2+$ : diameter $>$ half of histamine diameter, but < histamine diameter; $3+$ : diameter = histamine diameter; $4+$ : wheal with pseudopod

of wheal reactions [(the longest wheal diameter + sum of the longest orthogonal lines radiating from both sides of the first line)/2] were measured and recorded. Wheal diameters $>3 \mathrm{~mm}$ were considered positive and categorised as $0-4+(0$ : negative; 1+: diameter less than half of histamine diameter; $2+$ : diameter more than half of but less than histamine diameter; $3+$ : diameter equal to that of histamine; 4+: wheal with pseudopod).

All data analysis was done using the Statistical Package for the Social Sciences version 11.0 (SPSS Inc, Chicago, IL, USA). The $t$-test was used for comparing means, Mann-Whitney $U$ test for comparing medians, chi-square or Fisher's exact test for comparing proportional data, and linear or logistic regression for testing correlation.

\section{RESULTS}

A total of 100 volunteers were enrolled in the study, and their baseline characteristics are summarised in Table I. The mean age of the volunteers was 28 (range 19-59) years and 58\% were female. Of the 100 volunteers, 19 had a positive family history of allergic disease.

All the 100 volunteers had a positive response to histamine, with a mean wheal diameter of $5.24 \mathrm{~mm}$, regardless of age, gender, family history of atopic disease and results of the skin prick test. There was no association between positive skin test among healthy volunteers and diameter of the histamine wheal.

In all, 42 volunteers had a positive skin prick test for at least one allergen. The median number of sensitised allergens was 2 (range 1-7). Those who had positive skin tests were younger than those with negative skin tests (mean age 25.5 vs. 29.2 years; $\mathrm{p}<0.05)$. The group with positive skin tests had a higher proportion of males $(57.1 \%$ vs. $31.0 \%$; $p<0.01)$ and first-degree relatives with a history of atopic diseases $(31.0 \%$ vs. $10.3 \%$; $\mathrm{p}<0.05)$ than those with negative skin tests. The most common sensitised allergens in healthy asymptomatic volunteers were mite $(\mathrm{n}=33)$, house dust $(\mathrm{n}=23)$ and American cockroach $(n=20)$, as shown in Table II. There was a total of 19 volunteers who had a positive response to more than three allergens, with the two most common allergens among this subgroup being mite and house dust.

\section{DISCUSSION}

Several studies have found that $3 \%-50 \%$ of healthy individuals may have a positive allergen skin test, and this incidence varies according to ethnicity, region, method of testing and type of reagents used. ${ }^{(5,6,12-16)}$ In the present study, the incidence of healthy volunteers who were found to be sensitised to aeroallergens was $42.0 \%$; this is within the reported range. Compared to a Thai study conducted by Daengsuwan et al, a larger proportion of volunteers in our study population was sensitised to common aeroallergens than their healthy controls $(42.0 \%$ vs. $35.2 \%) ;{ }^{(9)}$ however, the prevalence of positive skin prick tests in our healthy volunteers was similar to that in their study's adult atopic and asthma group (42.0\% vs. $43.7 \%) .{ }^{(9)}$ This trend of increasing prevalence of positive skin tests among healthy Thai volunteers may suggest exposure to a greater number of indoor allergens in an urban environment, with the most common allergens being mite and house dust. The tropical 
climate of Thailand, with high humidity all year round, could be a contributing factor. As living in urban areas reduces one's exposure to endotoxins, urban dwellers are thus more prone to developing atopy, ${ }^{(17)}$ which could account for the prevalence of allergic sensitisation in this study population.

In the current study, one of the associated factors of sensitisation to aeroallergens was a family history of atopic disease in first-degree relatives, with a higher prevalence of positive skin test in healthy volunteers compared to those with negative skin test $(31.0 \%$ vs. $10.3 \%$; $<0.05)$. This finding confirms the report by Crestani et al that children of atopic parents react to a higher number of sensitised allergens. ${ }^{(18)}$ The notion that a family history of atopy plays a role in the development of skin test sensitisation in first-degree relatives is not new, since atopic disease is known to have a genetic predisposition.

Although a significant body of knowledge has suggested that a person with positive skin test who is sensitised to aeroallergens would be more prone to developing allergic disease, whether an asymptomatic individual who has skin test reactivity would have atopic disease in the future is beyond the scope of our study. Hagy and Settipane reported that $32 \%$ of healthy subjects who tested positive to common aeroallergens subsequently developed allergic rhinitis, while $6 \%$ of them developed asthma during the seven-year follow-up period. ${ }^{(6)}$ Similarly, Bodtger et al's study found that $6 \%$ of asymptomatic patients who were found to be sensitised to bird pollen would develop pollen allergy after three years. ${ }^{(5)}$

Knowledge of the common sensitised allergens found among the population in a region could assist medical practitioners in narrowing down the panel of allergens tested in daily practice. In allergy skin tests, a smaller panel with three or four of the most common allergens would be more cost effective. We found a low rate of sensitisation to grass and moulds in our study population, and thus it may be practical to exclude the use of these reagents in routine skin tests.

Our study is, however, not without its limitations. Firstly, the study population, which represents urban-dwelling people, is not representative of the whole population living in the region. Secondly, the panel of allergens used in the skin prick test was limited to 16 common allergens, which may not cover the sensitised allergens profile of any given individual. Lastly, as the skin prick test in asymptomatic individuals presents some limitations in identifying false positive cases, it should only be indicated in atopic symptomatic individuals.
In conclusion, up to $42 \%$ of healthy volunteers, particularly individuals with a family history of atopy, were found to be sensitised to common allergens in our study. Reactivity of the skin test without the symptoms of allergy is not indicative of allergic disease. Therefore, skin tests should only be indicated in symptomatic individuals with atopy.

\section{REFERENCES}

1. Keeley DJ, Neill P, Gallivan S. Comparison of the prevalence of reversible airways obstruction in rural and urban Zimbabwean children. Thorax 1991; 46:549-53.

2. Ng'ang'a LW, Odhiambo JA, Mungai MW, et al. Prevalence of exercise induced bronchospasm in Kenyan school children: an urban-rural comparison. Thorax 1998; 53:919-26.

3. Van Niekerk $\mathrm{CH}$, Weinberg EG, Shore SC, Heese HV, Van Schalkwyk J. Prevalence of asthma: a comparative study of urban and rural Xhosa children. Clin Allergy 1979; 9:319-4.

4. Yemaneberhan H, Bekele Z, Venn A, et al. Prevalence of wheeze and asthma and relation to atopy in urban and rural Ethiopia. Lancet 1997; 350:85-90.

5. Bodtger U, Poulsen LK, Malling HJ. Asymptomatic skin sensitization to birch predicts later development of birch pollen allergy in adults: a 3-year follow-up study. J Allergy Clin Immunol 2003; 111:149-54.

6. Hagy GW, Settipane GA. Risk factors for developing asthma and allergic rhinitis. A 7-year follow-up study of college students. J Allergy Clin Immunol 1976; 58:330-6.

7. Horak F. Manifestation of allergic rhinitis in latent-sensitized patients. A prospective study. Arch Otorhinolaryngol 1985; 242:239-45.

8. Choovivathanavanich P, Oshima S, Miyamoto T, Kanthavichitra N, Suwanprateep P. Mite sensitivity studies on Dermatophagoides farinae and house-dust allergy in Thai subjects. J Med Assoc Thai 1971; 54:826-35.

9. Daengsuwan $T$, Lee BW, Visitsuntorn $N$, et al. Allergen sensitization to aeroallergens including Blomia tropicalis among adult and childhood asthmatics in Thailand. Asian Pac J Allergy Immunol 2003; 21:199-204.

10. Prasarnphanich T, Sindhurat S. Sensitization to common indoor allergens and its association with allergic diseases in Thai female high-school students. Pediatr Allergy Immunol 2005; 16:402-7.

11. Kongpanichkul A, Vichyanond P, Tuchinda M. Allergen skin test reactivities among asthmatic Thai children. J Med Assoc Thai 1997; 80:69-75.

12. Bodtger U. Prognostic value of asymptomatic skin sensitization to aeroallergens. Curr Opin Allergy Clin Immunol 2004; 4:5-10.

13. Kerkhof M, Schouten JP, De Monchy JG. The association of sensitization to inhalant allergens with allergy symptoms: the influence of bronchial hyperresponsiveness and blood eosinophil count. Clin Exp Allergy 2000; 30:1387-94.

14. Leung R, Ho P. Asthma, allergy, and atopy in three south-east Asian populations. Thorax 1994; 49:1205-10.

15. Norrman E, Rosenhall L, Nystrom L, Jonsson E, Stjernberg N. Prevalence of positive skin prick tests, allergic asthma, and rhinoconjunctivitis in teenagers in northern Sweden. Allergy 1994; 49:808-15.

16. Sin A, Kose S, Terzioglu E, et al Prevalence of atopy in young healthy population, in Izmir, Turkey. Allergol Immunopathol (Madr) 1997; 25:80-4.

17. Heederik D, von Mutius E. Does diversity of environmental microbial exposure matter for the occurrence of allergy and asthma? J Allergy Clin Immunol 1991; 130:44-50.

18. Crestani E, Guerra S, Wright AL, Halonen M, Martinez FD. Parental asthma as a risk factor for the development of early skin test sensitization in children. J Allergy Clin Immunol 2004; 113:284-90. 